\title{
L’abbé Louis-François Laflèche, missionnaire dans l’Ouest
}

\section{Nive Voisine}

Volume 37, 1970

La vie religieuse au Manitoba

URI : https://id.erudit.org/iderudit/1007276ar

DOI : https://doi.org/10.7202/1007276ar

Aller au sommaire du numéro

Éditeur(s)

Les Éditions Historia Ecclesiæ Catholicæ Canadensis Inc.

ISSN

0318-6172 (imprimé)

1927-7067 (numérique)

Découvrir la revue

Citer cet article

Voisine, N. (1970). L'abbé Louis-François Laflèche, missionnaire dans l'Ouest. Sessions d'étude - Société canadienne d'histoire de l'Église catholique, 37, 61-69. https://doi.org/10.7202/1007276ar

Tous droits réservés @ Les Éditions Historia Ecclesiæ Catholicæ Canadensis Inc., 1972
Ce document est protégé par la loi sur le droit d'auteur. L'utilisation des services d'Érudit (y compris la reproduction) est assujettie à sa politique d'utilisation que vous pouvez consulter en ligne.

https://apropos.erudit.org/fr/usagers/politique-dutilisation/ 


\section{L'abbé Louis-François Laflèche, missionnaire dans l'Ouest}

$\mathrm{M}^{\mathrm{gr}}$ Louis-François Laflèche, deuxième évêque de Trois-Rivières, a été dans la deuxième moitié du $\mathrm{XIX}^{\mathbf{e}}$ siècle, une des personnalités les plus importantes du Québec. Comme évêque, "il n'y a pas un homme qui ait tant fait parler de lui dans tout l'épiscopat de cette province ${ }^{1}$. Disciple de $\mathbf{M}^{\mathrm{gr}}$ Ignace Bourget, de Montréal, il a incarné pendant longtemps l'idéal ultramontain et a dirigé les forces antilibérales dans des combats demeurés célèbres: la querelle de l' " influence indue ", la bataille autour de l'Université Laval, la controverse au sujet de la division du diocèse de Trois-Rivières, les luttes à propos de l'éducation, et que sais-je encore. Orateur éloquent qu'on venait entendre de partout, il a été de toutes les fêtes et démonstrations patriotiques et religieuses du Canada français. Ce qui ne l'a pas empêché d'être, dans son diocèse, un administrateur bien coté, un initiateur de fondations religieuses diverses et un éducateur de son peuple.

Mais avant d'être ce "défenseur de la cité ", cet intrépide qui avait en lui " du soldat et du chevalier » et dont le nom « était synonyme de vaillance, de courage ${ }^{2}$ ", Louis-François Laflèche a été un modeste professeur de collège et surtout, pendant 12 ans, un humble missionnaire dans les plaines de l'Ouest canadien. C'est de ce dernier aspect de sa vie - un peu moins connu que les autres - que je vous entretiendrai en vous rappelant ce qui l'a poussé à devenir missionnaire, en décrivant son activité apostolique et en vous signalant comment s'est terminée son aventure dans l'Ouest.

\section{1 - La vocation de missionnaire}

Louis-François Laflèche est né à Sainte-Anne-de-la-Pérade le 4 septembre 1818. Son père, Louis-Richer-Laflèche, vit modestement et frugalement sur la terre que lui a léguée son père et qui est située tout près de l'église, à côté du magasin de Pierre-Antoine Dorion, député "libéral " de Champlain et père des futurs leaders " rouges" Antoine-Aimé et Jean-Baptiste Dorion. Marie-Anne Joubin-Boisvert, sa mère, a étudié chez les Ursulines et elle peut lui donner de bonne heure les premiers rudiments de connaissances. Son grand-père, Modeste Richer-Laflèche, maître-chantre à Sainte-Anne, apprend au jeune homme

1 Le Soleil, 15 juillet 1898.

2 Ces expressions sont tirées de l'éloge fait par $\mathrm{M}^{\mathrm{gr}}$ Bruchési aux funérailles de Laflèche. Arthur S AVA ÈTE, Voix canadiennes, Vers l'abime, t. VI, M ${ }^{\text {gr Laflèche }}$ et la Division du Diocèse des Trois-Rivières, Paris, Savaète, (c1912), p. 552. 
le plain-chant. Surtout un instituteur qui pensionne chez les Laflèche, Craig Morris, lui enseigne à coups de fërule mais efficacement tout ce qu'il sait lui-même. Ce qui fait que le jeune Laflèche est un privilégié et qu'il peut entrer au Collège de Nicolet pour faire son cours classique.

Pendant ses études secondaires, Laflèche se révèle déjà un esprit discipliné et avide de science et un excellent élève. Ce n'est donc une surprise pour personne de le voir choisir l'état ecclésiastique en 1838. Pour se préparer au sacerdoce, il n'a même pas à changer de maison; c'est au Collège de Nicolet qu'il s'initie à la théologie tout en exerçant la tâche de professeur et, pendant un an, celle d'économe. Il semble bien qu'il se prépare à faire carrière au service de son Alma Mater.

C'est dans ce milieu que naît sa vocation missionnaire. Le Séminaire de Nicolet n'est pas seulement un collège select, gratifié d'une charte royale et logé dans des édifices imposants; il est surtout un des principaux pourvoyeurs de missionnaires pour l'Ouest canadien. Les deux premiers prêtres envoyés à la Rivière-Rouge par le diocèse de Québec étaient des anciens de Nicolet: Jean-Norbert Provencher et Sévère Dumoulin. Plusieurs qui les suivirent venaient de la même source, tels les abbés Arsène Mayrand, Georges-Antoine Belcourt et Jean Harper. Un lien très fort s'établit bientôt entre les missions de l'Ouest et Nicolet. Les anciens missionnaires viennent raconter leur aventure aux étudiants dans l'espoir d'éveiller des vocations; $M^{p 1}$ Provencher, lors de ses fréquents voyages dans l'Est, fait de longs séjours au collège - sa famille est d'ailleurs de Nicolet - et il s'entretient volontiers avec les élèves et les professeurs. Les besoins de la lointaine Rivière-Rouge sont donc bien connus de la communauté de Nicolet.

Laflèche n'échappe pas à la règle. En octobre 1843, alors qu'il n'est encore que diacre, il a de longues conversations avec $\mathrm{M}^{\mathrm{gr}}$ Provencher, venu une fois de plus solliciter de l'aide pour sa jeune Église. Le jeune lévite l'écoute décrire le vaste champ d'apostolat confié à sa responsabilité et ce qu'il pourrait y récolter s'il avait des missionnaires en plus grand nombre. Il est peut-être frappé par le ton mélancolique de l'évêque qui lui confie: "Je ressemble à un chêne qui demeure seul debout au milieu d'une plaine où l'orage emporte tous les autres arbres ${ }^{3}$." A-t-il été, en ce moment, touché par l'exemple d'un courage si indomptable? A-t-il senti monter en lui le goût de l'aventure ? Nous ne pouvons le dire avec certitude, car Laflèche ne nous a pas laissé de témoignages sur son état d'âme. Mais nous savons qu'il décide aussitôt de s'offrir à accompagner $\mathrm{M}^{\mathrm{gr}}$ Provencher. Il abandonne immédiatement sa chaire de rhétorique et se rend à Québec pour se

3 Dom Paul Benô̂t, Vie de $M^{g r}$ Taché, archevêque de St-Boniface, Montréal, Beauchemin, 1904, t. 1, p. 53. 
faire ordonner le 4 janvier 1844 et se préparer à partir pour SaintBoniface au printemps.

En plus de l'influence du milieu nicolétain, il faut noter le rôle de la mère de l'abbé Lafièche. Elle avait du sang indien dans les veines, car elle descendait d'une Indienne de l'Ouest et de Louis-Joseph Gastineau, troisième seigneur du fief Sainte-Marie. En lui parlant de sa grand-mère, elle a suscité très tôt chez son fils Louis-François un intérêt profond pour les plaines de l'Ouest. Chrétienne convaincue, elle lui a inculqué un goût de la religion et du dévouement, une piété sincère, un sens du devoir, un amour du prochain dont il lui sera gré toute sa vie. Qui sait ? C'est probablement sur les genoux de cette mère vénérée que s'est préparée la vocation missionnaire du futur évêque. Et en 1843, le jeune diacre a peut-être connu une poussée du sang indien qui coulait en ses veines.

Quoi qu'il en soit, Louis-François Laflèche décide de se consacrer aux missions de l'Ouest et il abandonne son collège et ses parents pour commencer une vie nouvelle dans ces terres lointaines.

\section{2-Son activité missionnaire}

Laflèche part pour Saint-Boniface en avril 1844. Après quelques jours d'attente à Trois-Rivières et à Montréal, il s'embarque à Lachine le 27 avril. Il fait le voyage en compagnie du gouverneur George Simpson et de $\mathrm{M}^{\mathrm{gr}}$ Provencher, de l'abbé Joseph Bourassa, nouveau missionnaire comme lui, et de quatre Sœurs Grises destinées aux missions de la Rivière-Rouge. La randonnée est longue et pénible: elle dure 55 jours remplis de difficultés (les 72 portages par exemple), d'accidents (la sœur Lagrave, "volumineuse et pesante ", se fait une douloureuse entorse et doit être transportée sur un brancard par deux solides Iroquois), de tempêtes. Laflèche s'adapte assez bien à ce genre de vie pittoresque et il le décrira avec un certain enthousiasme ${ }^{4}$, mais il contracte un rhumatisme virulent qui l'handicapera pendant tout son séjour dans l'Ouest et finalement l'obligera à revenir dans son pays natal.

Sur le coup, cette maladie n'inquiète personne. Dès le mois de juillet 1844, $\mathrm{M}^{\mathrm{gr}}$ Provencher écrit à $\mathrm{M}^{\mathrm{gr}}$ Bourget: « $\mathrm{M}$. Laflèche a pris force médecines et il est bien ${ }^{5}$. " 0 n peut donc compter sur lui pour le ministère apostolique.

Sa première tâche sera de desservir Saint-François-Xavier de la Prairie du Cheval Blanc. Il exerce son apostolat auprès des Blancs

4 Louis-François Laflèche, \& Des Trois-Rivières à Saint-Boniface en 1844 ,, Les Cloches de Saint-Boniface, XXIV, 2 (février 1925), p. 31-33.

$5 \mathrm{M}^{\mathrm{gr}}$ Provencher à $\mathrm{M}^{\mathrm{gr}}$ Bourget, 30 juillet 1844 , Les Cloches de SaintBoniface, XVIII, 19-20 (1-15 octobre 1919), p. 263. 
et des Métis, car il ne connaît pas suffisamment les langues indiennes pour "rendre aucun service important à ces pauvres sauvages qui se trouvent sans pasteurs ${ }^{6}$ \%. Cette fonction curiale, Laflèche la gardera jusqu'au printemps 1845 ; il la reprend à partir de 1849 au retour de sa mission de l'Île-à-la-Crosse et il l'exerce jusqu'à son départ pour le Canada en 1856. C'est à ce titre de "curé » qu'il accompagne ses ouailles métisses de Saint-Boniface et de Saint-François-Xavier à la chasse au bison en 1850 et en 1851. Cette dernière année, il frôle la mort de près, car le parti est attaqué par une bande de Sioux. Les assaillants, beaucoup plus nombreux que les Métis, encerclent leur campement: bien décidés à vendre chèrement leur vie, les Métis organisent un rempart et attendent l'attaque. En surplis blanc et le crucifix à la main, Laflèche se montre partout dans le camp retranché et exhorte tout le monde au courage. Lui-même affronte le combat sans peur. Et le miracle arrive; après plusieurs attaques coûteuses, les Sioux abandonnent la partie et se retirent en disant: "Vous avez avec vous un Manitou qui vous défend. " Laflèche commente ainsi l'événement à son confrère l'abbé Thomas Caron, de Nicolet: "Qu'en dites-vous, mon ami, n'est-ce pas là joliment faire le général ? Et si le défunt Bonaparte m'eût entendu, ne m'aurait-il pas, en passant, jeté quelque croix de Légion d'Honneur ${ }^{7}$ ? " Mais il ne faudrait pas restreindre l'apostolat de Laflèche à cet exploit guerrier; il administrait les sacrements et il s'occupait activement des œuvres d'éducation et de charité de $\mathrm{M}^{\mathrm{gr}}$ Provencher.

Cependant, Laflèche était venu dans l'Ouest surtout pour se consacrer à l'évạngélisation des nombreuses tribus indiennes des Plaines. C'est pourquoi, dès son arrivée, il s'attaque au principal obstacle qui l'éloigne de son but, l'ignorance de la langue. Pendant les premières années, il passe donc une bonne partie de son temps à l'étude du sauteux, du montagnais et surtout du cris. Tous les hivers y sont consacrés, que ce soit à l'évêché de Saint-Boniface en 1845-1846 ou à l'Île-à-la-Crosse l'année suivante. Ses maîtres sont des missionnaires plus anciens déjà rompus à la pratique de ces langues (l'abbé Belcourt par exemple) ou même des Indiens délurés. Laflèche travaille avec un enthousiasme juvénile qui porte fruit, puisqu'en 1849 il peut publier une étude sur la langue crise.

Le 8 juillet 1846, Laflèche part enfin pour son premier poste de mission, l'île-à-la-Crosse. C'est un endroit isolé à $56^{\circ} 25^{\prime}$ de latitude nord, une espèce de butte de sable entourée de marais et de lacs; mais

$6 \quad$ Ibid., p. 265.

7 L.F. Laflèche à Thomas Caron. 4 septembre 1851, cité par S4vak̀te, Voix canadiennes, Vers l'abime, t. X. Vie de $M^{g r}$ L.F. Laflèche, ses contrariétés et ses Euvres, Paris, Savaète, (s.d.), p. 181-183. 
c'est aussi le centre d'un immense district où errent des groupes de Montagnais et de Cris au nombre d'environ 2,000 âmes. Peu éloignée du Portage La Loche, grand rendez-vous des brigades de la rivière Mackenzie et de celles du Fort Garry, l'Île-à-la-Crosse se trouve par conséquent sur la grande voie de l'Ouest et du Nord et la Compagnie de la Baie d'Hudson y a construit un fort, c'est-à-dire quelques maisons et des hangars entourés d'une palissade, qui sert de lieu de rassemblement aux Indiens. Laflèche s'y installe avec un compagnon qui deviendra presque aussi célèbre que lui, le père Antonin-Alexandre Taché, futur archevêque de Saint-Boniface.

Après un hiver passé à étudier les langues indiennes, les deux missionnaires se partagent la tâche apostolique. Le père Taché se charge des courses auprès des Indiens et il va au loin jeter les bases de nouvelles missions. Laflèche, que la maladie a repris, fait du ministère auprès des Indiens qui résident dans l'entourage du fort ou qui y passent. Il fait de la prédication avec une éloquence déjà remarquable et il s'efforce de leur expliquer la doctrine catholique et de leur apporter le secours de la religion. Plus sédentaire que nomade, il s'adonne à tous les métiers: charpentier, menuisier, jardinier, cuisinier, et il est toujours là pour accueillir son confrère au retour de ses courses lointaines.

Dès 1848, Laflèche souffre de plus en plus d'un rhumatisme dans une jambe et dans un bras; sous l'effet de l'humidité des marécages, celui-ci " se changea en bosses, puis en plaies aussi incommodes que pénibles ${ }^{8}$. Malgré tous les soins du père Taché, la maladie augmente et oblige Laflèche à abandonner ses projets d'expéditions. Comme le dit son compagnon: "La maladie de Laflèche et une plus grande aptitude pour tout ce qu'exigeait le soin d'une mission établie, lui commandant une espèce d'inamovibilité, les délices de la promenade revenaient de droit à son compagnon ${ }^{9}$." Le malade lui-même prend la chose calmement et, plus tard, remarque malicieusement: "Pour me punir de ma paresse, le Bon Dieu m'envoya un rhumatisme qui me tourmenta longtemps, et pour m'empêcher d'oublier la leçon, il a eu soin, en le retirant, de me laisser boiteux ${ }^{10}$. " Cette infirmité est néanmoins un obstacle majeur pour le missionnaire et elle affaiblit l'efficacité de l'équipe, qu'il faut renforcer par l'addition d'un nouveau compagnon. le père Henri Faraud, qui arrive à l'île-à-la-Crosse en juillet 1848.

Laflèche quitte cette mission en 1849 , non pas tant à cause de sa maladie (des médecins qui ont visité le missionnaire disent que le

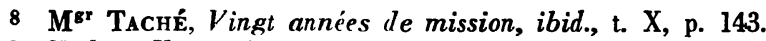

9 Ibid., t. X, p. 145.

10 Ibid., t. X, p. 143. 
mal n'est pas dangereux) que parce qu'il est rappelé à Saint-Boniface par $M^{\text {gr }}$ Provencher qui veul en faire son coadjuteur et son successeur. Même si le projet tourne court, Laflèche demeure à la Rivière-Rouge jusqu'en 1856 et participe de plus en plus aux activités de la colonie. En 1850, il est nommé au Conseil d'Assiniboia; il est assermenté à la réunion au 5 septembre. Les procès-verbaux indiquent qu'il joue un rôle très important à chaque séance: c'est souvent lui qui prend l'initiative de telle ou telle résolution, il est régulièrement désigné pour faire partie des comités (celui qui étudie l'organisation d'un bac sur les rivières Rouge et Assiniboine, celui qui doit conseiller sur l'achat d'une presse typographique, celui qui doit étudier l'état des lois, etc.). Ses interventions concernent tout aussi bien l'éducation que la chasse aux loups, la vente des propriétés immobilières, l'adoption des comptes publics et le prix de transport des journaux. Sa dernière demande a pour objet la nomination d'un arpenteur qui puisse comprendre le français pour traiter avec les "Canadiens ${ }^{11}$ ". Sous la sécheresse des comptes rendus, on décèle le désir d'aider la population qu'il dessert au point de vue spirituel; pour Laflèche, sa fonction de conseiller est le prolongement de sa tâche apostolique.

\section{3 - La fin du séjour}

Venu dans l'Ouest pour évangéliser les Indiens, Laflèche fait donc beaucoup de " métiers ". Il les accepte volontiers, car il pense contribuer ainsi à la gloire de Dieu. Il y a cependant une charge qu'il repousse avec un acharnement constant, c'est l'épiscopat.

Dès $1848, \mathrm{M}^{\mathrm{gr}}$ Provencher décide d'en faire son successeur. Il écrit aux évêques du Québec et il explique son projet à $\mathbf{M}^{\mathrm{gr}}$ Bourget:

Je sens que devenu vieux, je ne puis pas facilement visiter les missions éloignées, mais elles ne font que commencer et il n'y a pas encore de chrétiens pour la peine. [...] Je pense, depuis que je suis séparé de Québec, à me pourvoir d'un coadjuteur jeune, qui pourrait courir au loin, pendant que j'expédierais la besogne ici. [...] J'avais proposé $M$. Laflèche comme le plus capable de remplir cette place. Il est bien instruit dans toutes les sciences qu'on enseigne dans les collèges. Il est studieux, d'un excellent caractère, sans prétention, il ne sait pas ce qu'il est à en juger par sa conduite. Je l'ai amené dans cette intention, si je vivais assez pour lui donner le temps d'acquérir l'âge requis. Il a vingt-neuf ans. Il parle passablement l'Anglais, entend le Grec et est un peu initié aux langues sauteuse, crise et montagnaise. Devenu pasteur, il pourra se faire entendre de presque toutes ses brebis ${ }^{12}$.

La nomination tarde car, à Québec, on trouve Laflèche trop jeune. Finalement, $M^{\mathrm{gr}}$ Provencher, appuyé par les évêques de Québec et de

11 E. H. OuvER, éd., Le Nord-Ouest canadien, son évolution primitive et ses archives législatives, Ottawa, Imprimeur du gouvernement, 1916, t. l, p. 236-276.

$12 \mathrm{M}^{\mathrm{gr}}$ Provencher à $\mathrm{M}^{\mathrm{gr}}$ Bourget, 18 juin 1847, Les Cloches de SaintBoniface, XX, 2 (15 février 1921), p. 31s. 
Montréal, demande en 1849 des lettres testimoniales pour son protégé. Le 20 octobre, de Naples où il a dû se réfugier, Pie IX nomme l'abbé Laflèche évêque d'Arath et coadjuteur de Provencher. Mais tout n'est pas réglé, car il faut compter encore avec l'entêtement du missionnaire.

Laflèche soulève, par humilité, les objections d'usage en pareille circonstance, mais il insiste surtout sur son mauvais état de santé. A ses yeux, son infirmité est un empêchement dirimant: "Vous voulez un coadjuteur vigoureux et je suis infirme; vous avez besoin d'un coadjuteur qui puisse parcourir à votre place ces immenses régions et je suis plus incapable de voyager que vous ${ }^{13}$." Par un heureux " hasard ", son mal empire dès qu'on lui propose l'épiscopat. $M^{\text {gr }}$ Provencher le note avec lucidité: " Il se complaît dans ses infirmités parce qu'elles l'empêchent d'être évêque. charge qu'il aurait été difficile de lui faire accepter ${ }^{14}$." L'évêque se résigne donc à ne pas compter sur Laflèche et il fait désigner à sa place le père Antonin-Alexandre Taché.

La maladie et la lassitude qui en découle obligent enfin Laflèche à quitter l'Ouest pour son pays natal. Il le fait une première fois en 1854 dans le double but de se reposer et de régler certaines affaires de famille. Ce n'est qu'un voyage de quelques mois, car il a promis à $\mathrm{M}^{\mathrm{gr}}$ Taché de revenir. D'ailleurs, celui-ci a pris soin d'écrire à l'évêque de Québec: "Je regarde la présence de $M$. Laflèche comme indispensable au bien, et ce serait une peine bien sensible pour moi s'il ne revenait pas, mais il reviendra ${ }^{15}$."

Laflèche retourne en effet à la Rivière-Rouge en mai 1855, non sans avoir rédigé pour le Rapport sur les Missions du diocèse de Québec une étude sur le diocèse de Saint-Boniface. Il fait ce travail avec une certaine répugnance, car il n'a pas ses notes et il n'aime guère " ces sortes de relations". Il réussit quand même à décrire avec précision cet immense diocèse, l'un "des plus grands du monde ", qui s'étend de la Rivière-Rouge aux Rocheuses et de la frontière américaine à la "mer glaciale". Il consacre plusieurs pages aux Indiens; règle générale, il n'est pas tendre pour les tribus (surtout celles des plaines) où. dit-il, « l'homme est descendu au dernier degré de l'échelle humaine ", dans un "état de dégradation et de méchanceté inouï ". Enfin, il analyse chacune des missions qu'il a connues. Il décrit succinctement la paroisse de Saint-Boniface, résidence ordinaire de l'évêque: la " paroisse de la Rivière Sale" (futur Saint-Norbert), encore desservie de Saint-Boniface; la paroisse de Saint-François-Xavier du Cheval

13 Savaète, op. cit., t. X, p. 339.

$14 \mathrm{M}^{\mathrm{gr}}$ Provencher à $\mathrm{M}^{\mathrm{gr}}$ Bourget, 29 novembre 1849, Les Cloches de SaintBoniface, XX, 8 (août 1921), p. 152.

$15 M^{\mathrm{gr}}$ Tassé à $\mathrm{M}^{\mathrm{gr}}$ Turgeon, 13 novembre 1854, Benô̂T, op. cit., t. 1, p. 288. 
Blanc d'où l'on rayonne vers la rivière Esturgeon et le lac Manitoba; les résidences du lac Sainte-Anne, de Saint-Jean-Baptiste de l'Île-à-laCrosse, d'Athabasca et du lac La Biche. Quatre prêtres séculiers y travaillent - ce sont les abbés Albert Lacombe (il deviendra plus tard oblat), Jean-Baptiste Thibault, Joseph Bourassa et lui-même - en compagnie de huit pères oblats: François-Xavier Bermond, Jean Tissot, Valentin Végréville, Henri Faraud, René Rémas, Henri Grollier, Augustin Maisonneuve et $\mathrm{M}^{\mathrm{gr}}$ Alexandre Taché ${ }^{16}$. Le texte laisse supposer que Laflèche continuera à travailler dans ce diocèse de mission, mais dès juin 1856 il part définitivement pour le Canada " allant demander à sa terre natale une santé épuisée par douze années d'un généreux dévouement ${ }^{17}$ ".

\section{Conclusion}

L'œuvre missionnaire de Laflèche a été diversement appréciée. Les critiques n'ont pas manqué. L'auteur de The Rainbow in the North (probablement $\mathrm{M}^{11 \mathrm{e}} \mathrm{S}$. Tacker) accuse les missionnaires de l'île-à-laCrosse de baptiser précipitamment les Indiens: "Au lieu d'instruire les candidats au baptême, on leur donnait un papier contenant le nom des patriarches et des apôtres, la représentation du ciel, des saints, du déluge, du temple de Salomon, etc. ${ }^{19}$. "Le libéral Vieux-Rouge reproche à Laflèche sa méthode trop autoritaire: "Comme autrefois les papes guerriers, il brandissait le crucifix comme si c'eût été un glaive. Il se montrait autoritaire comme un évêque espagnol, et si vous avez lu ses lettres d'alors, vous remarquerez que sa meilleure narration est une description d'une bataille ${ }^{19}$."

Ces jugements trahissent trop les rivalités d'une secte ou d'un parti politique pour que nous les retenions. Nous leur préférons le témoignage déjà cité de $\mathrm{M}^{\mathrm{gr}}$ Provencher qui connaissait beaucoup plus intimement Laflèche. D'ailleurs, pendant les quelques années où il travaille dans les missions proprement dites, le futur évêque de TroisRivières n'a pas le temps d'innover. Comme les Pères Oblats qui œuvrent de plus en plus nombreux dans l'Ouest, il commence par se préparer à l'apostolat par l'étude des langues et des mœurs indiennes, puis, installé en un point stratégique, il contacte les Indiens et tâche de les préparer au Baptême. Il remplace le catéchuménat par une série d'instructions qui comportent l'enseignement des prières les plus usuelles:

16 Ls.F. Laflèche, * Mission de la Rivière-Rouge ", Rapport sur les Missions du diocèse de Québec, 11 (mars 1855), p. 118-137.

17 BENoîT, op. cit., t. 1, p. 339.

18 R.P. Morice, Histoire de l'Eglise catholique dans l'Ouest canadien du Lac Supérieur au Pacifique, t. 1, Montréal, Granger, 1921, p. 339.

19 VIEUX-Rouge, Les contemporains, Série de biographies des hommes du jour, $2^{\text {e }}$ livraison, Montréal, Filiatreault, 1899, p. 108. 
Pater, Ave, Credo, Gloria, des cantiques en langue indigène, des vérités fondamentales et des principaux devoirs du chrétien ${ }^{20}$. Enfin, comme les religieux, il se considère comme un membre d'une équipe qui travaille en commun.

Même si la maladie l'a empêché de réaliser tous ses rêves, les années passées dans l'Ouest ont fortement marqué le futur évêque de Trois-Rivières. Au physique, il conservera jusqu'à sa mort une légère claudication dont se gaussent facilement certains de ses adversaires, témoin cet abbé qui écrit: " $\mathrm{M}^{\mathrm{gr}}$ Laflèche commence à trembler sur sa patte croche (encore s'il n'y avait que la patte ${ }^{21}$ !). » Mais surtout il gardera un intérêt constant pour ce territoire; quelques années après son retour de l'Ouest, il songera même à y retourner, mais son évêque s'y opposera. Quand $\mathrm{M}^{\text {gr }}$ Taché viendra dans l'Est demander du secours et surtout des colons, Laflèche le secondera, mais, comme bien d'autres leaders canadiens-français de l'époque, il considérera l'émigration au Manitoba comme un pis-aller: "Ce qu'il y a de mieux pour un Canadien c'est de rester en son pays où il y a encore tant de bonnes terres couvertes de riches forêts et qui n'attendent que des hommes de cœur et de bonne volonté pour être mises en valeur. Quant à ceux que de pénibles circonstances forcent à laisser la province de Québec, c'est vers le Manitoba qu'ils doivent se diriger: là ils trouveront des terres toutes défrichées, de la plus grande fertilité et à des prix raisonnables, et surtout ils trouveront toutes les institutions du Canada pour l'éducation de leurs enfants et l'accomplissement de leurs devoirs religieux ${ }^{22}$. Enfin, il n'est pas besoin de rappeler la part qu'a prise Laflèche dans la question des écoles du Manitoba.

C'est jusqu'aux derniers instants de sa vie qu'il se souvint des belles années de son séjour dans l'Ouest. Il reposait déjà sur son lit de mort quand il demanda à voir une Indienne qui demeurait à Trois-Rivières et il s'entretint privément avec elle en langue indienne car, dit-il, c'est peut-être la dernière fois qu'elle pourra parler sa langue maternelle. Cette pieuse anecdote nous décrit bien ce qu'a été $\mathrm{M}^{\mathrm{gr}}$ Laflèche: un homme de cœur qui a été marqué par ses années de missions dans l'Ouest canadien.

Nive Voisine, prêtre,

Professeur à l'Institut d'Histoire, Université Laval, Québec.

20 Joseph-Etienne Champagne, Les Missions catholiques dans l'Ouest canadien, Ottawa, Editions de l'Université, 1949, p. 188-193. B3D30.

21 Calixte Marquis à $M^{\mathrm{gr}} \mathrm{D}$. Racine, 6 janvier 1884, ASTR, Fonds Laflèche,

22 Laflèche à l'abbé C.-A. Beaudry, 21 novembre 1888, Les Cloches de SaintBoniface, XXVIII, 10 (octobre 1929), p. 228s. 